\title{
A Practical 3D Measuring System of Parts Based on Laser Sensor
}

\author{
Sheng Feng Fang ${ }^{1, ~ a, ~ C a i ~ H o n g ~ S u, ~ b ~}{ }^{2,}$ Zhi Ye $\operatorname{Pan}^{3, c}$ \\ Ning Zhou Zhan ${ }^{4,}$, Bing He Wu ${ }^{5, e}$ \\ ${ }^{1}$ School of Automation, FoShan University, FoShan 528000, China \\ ${ }^{2}$ School of Automation, FoShan University, FoShan 528000, China \\ ${ }^{3}$ School of Automation, FoShan University, FoShan 528000, China \\ ${ }^{4}$ Kingsvision Automation Technology ( Foshan ) Co., Ltd. FoShan 528000, China \\ ${ }^{5}$ School of Automation, FoShan University, FoShan 528000, China \\ a641517050@qq.com, b168152620@qq.com, '735951851@qq.com \\ d514857817@qq.com, $871434621 @ q q . c o m$
}

Keywords: Laser Displacement Sensor Measuring system Triangulation Method 3D Imaging

Abstract: This paper had designed a 3D measuring system with low cost and high-precision. The 2D laser displacement sensor is the core component, and the Labview graphical programming software is the main software platform. PLC and stepping motor drives high-precision guides rail movement. Combining software and hardware to calibrate the system and establishing the transformation between the imaging point and the corresponding object point accurately, which obtain the 3D parameters of the measured object surface, completed the data collection, processing and preservation. Experiments demonstrate that the system can be metal, ceramic and other materials parts to achieve high-precision 3D measurement.

\section{Introduction}

At present most of the parts testing mainly uses the contact-type static measurement in the process of production and assembly. This measurement method is not suitable for soft materials high precision surface measurement. These factors such as speed, efficiency and accuracy are difficult to meet the requirements. And the measurement process is difficult to achieve automation and intelligence. With the continuous emergence of new optoelectronic products, the current foreign high precision detection has been widely used non-contact measurement methods, including the use of laser non-contact detection products more widely. But the domestic high-precision detection system almost all use foreign products or foreign technology products, and the prices of these goods are very expensive. Therefore, it is necessary to study the high precision laser detection products and relatively inexpensive equipment to improve product competitiveness.

For solving the above problems, a practical 3D measuring system for parts is developed. 2D laser displacement sensor is used to precisely control the movement of objects by high precision guide rails, and the system is calibrated by hardware and software. The transformation between imaging point and the object point is established, and the 3D 
topographic coordinates of the measured object surface are obtained. Experiments prove that the system can achieve high precision 3D measurement to metal, ceramic and other materials parts.

\section{System Designing}

\section{The principle of system}

The overall system framework is shown in figure 1
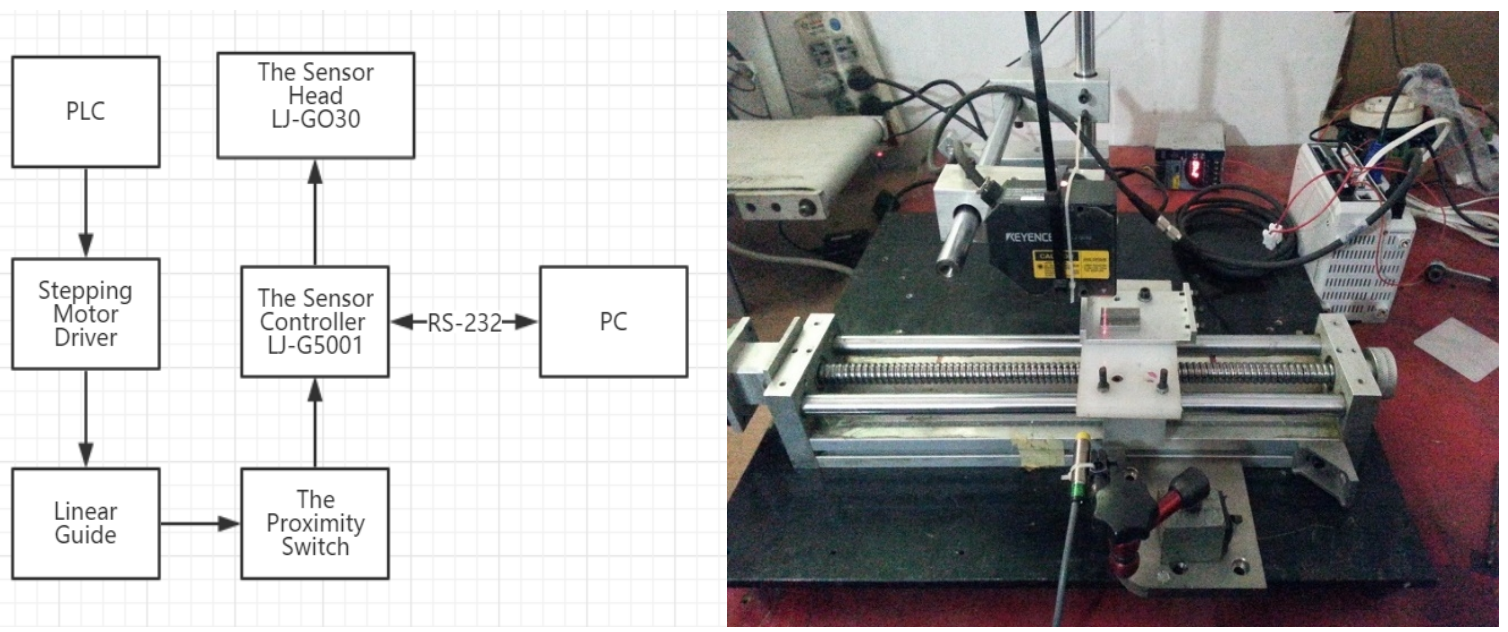

Fig.1 The overall system framework

The detection system consists of a sensor system, measuring platform, motion control system and software system. The main work process is that the part to be measured is placed on the slide which is moved by the linear guide. When the slider moves to the proximity switch, the PLC starts to trigger the 2D laser displacement sensor continuously, and shoots the sequence diagram.2D laser displacement sensor using light cutting method, and the photosensitive device (CMOS) receives the reflected light from the projected laser with band-shaped. The waveform of the reflected light is read for each vertical line of the captured image by using the triangulation method. which receives the height. And obtain the left and right position by taken data based on projection transformation method. The above process accurately establish the corresponding transformation between imaging points and object points. For this it will get the measured surface topography of 3D coordinates.

\section{Hardware designing}

Sensor system uses Keyence laser sensor LJ-G030 and controller LJ-G5001. The sensor measures the surface profile of the workpiece in the $\mathrm{X}$-axis and $\mathrm{Z}$-axis. Using direct measurement method.There are 28 measurement modes to measure the height, width and gap on the surface profile, with $3.8 \mathrm{~ms} * 4$ sampling period, and the diameter range of sensor measuring workpiece is $30 \mathrm{~mm}$. Measurement accuracy is $1 \mu \mathrm{m}$ on the $\mathrm{Z}$-axis (height) and $5 \mu \mathrm{m}$ on the $\mathrm{X}$-axis (width). The E3-CMOS image sensor in the controller LJ-G5001 provides stable measurements with a high sampling rate of $3.8 \mathrm{~ms}$ and a high accuracy of $0.1 \%$ of F.S. for measuring various heights, surface widths and clearances of the contours, interface area and so on. Motion control system consists of Mitsubishi PLC FX3GA-24MT and stepping motor 57BYGH246-2004A-8 and linear guides, position sensors.

\section{Software designing}

System software have two following parts, PC software design is the use of Labview, the program front panel as shown in Figure 2, program sequence chart is shown in figure 3, the front panel including the acquisition interface and analysis interface, the acquisition interface to read the image 
cloud point data, and the taken data being 3D modeling, while achieving real-time display, analysis interface can select any one profile with a cursor for more accurate analysis

The lower machine software uses the Mitsubishi PLC language programming, program sequence chart is shown in figure 4, Press the start button, the measured part by the rail at a certain speed of delivery, close to the material and the proximity switch to react, PLC start trigger sensor to measure a single contour of the object, and then get a series of data until the material left. At this point notify the upper computer to send contour storage commands, storage is completed and then stop the command, read the contour and the image data processing to obtain the desired height, position and other 3D information. Reading its data allows the 3D image to appear in the Labview front panel.

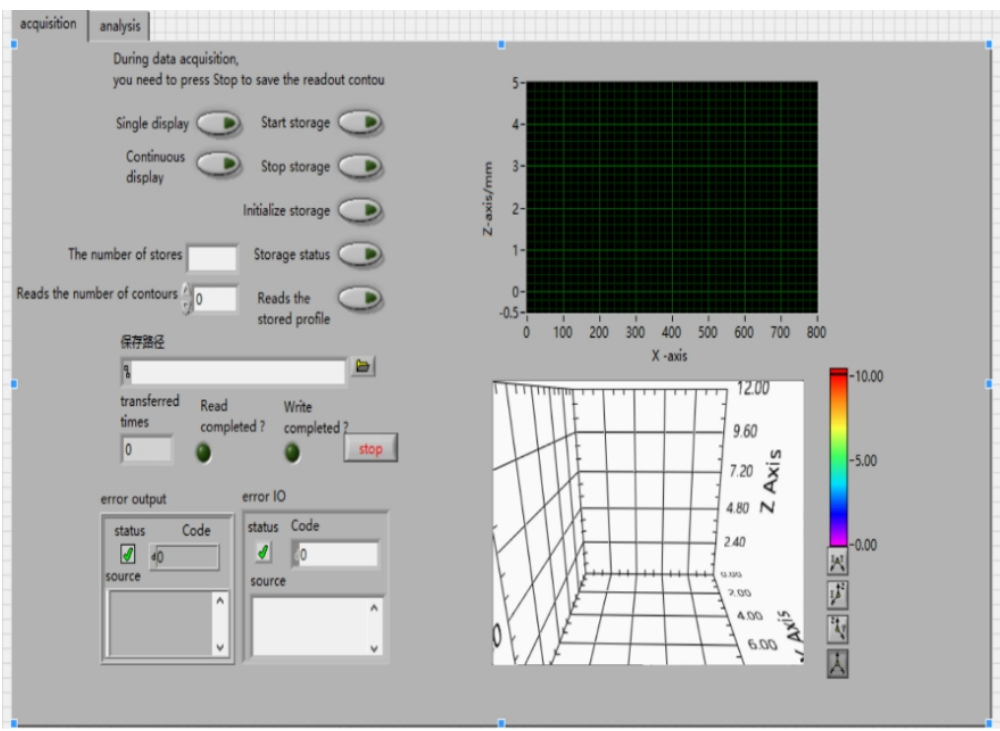

Fig. 2 The program front panel

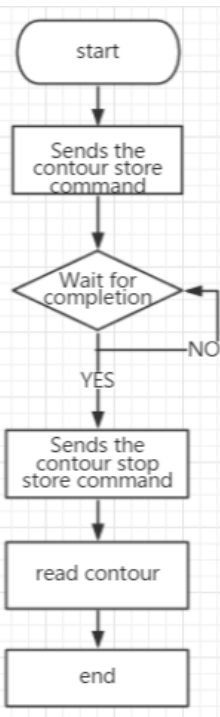

Fig.3 PC

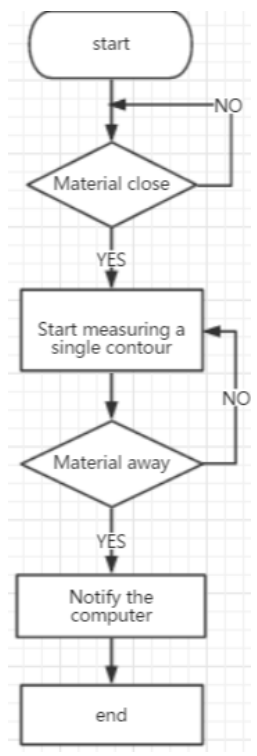

Fig.4 PLC

\section{Correction and calibration}

\section{Correction}

For eliminate the errors caused by the installation of the horizontal guide rail and the 2D laser sensor, the contour correction in software way should be carried out before the measurement. Horizontal correction is shown in figure 5 and figure 6 . The software used is LJ-Navigator by Keyence provided. The steps are as follows: (1). The system searches for the "Target profile" defined in the "Profile area" in the "Search area". (2). The system calculates the position difference in x-axis, z-axis, and tilt. (3). If the system finds an area that matches the contour area greater than or equal to the correlation value, the set area is adjusted and measured. If such a matching region is not found, the system will assume that the position correction fails,

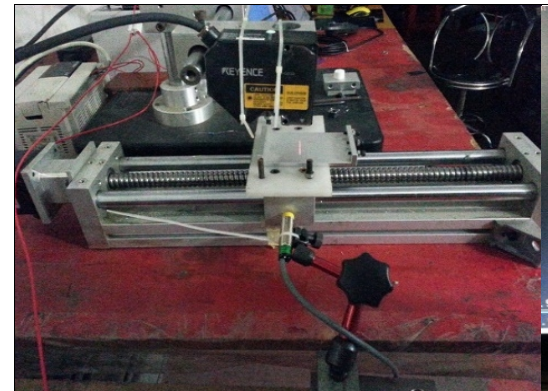

Fig.5 Practical operation of correction

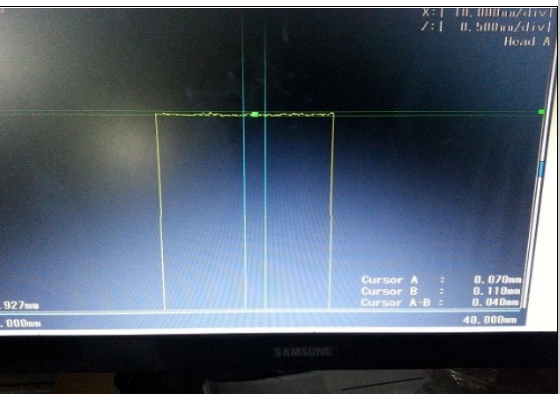

Fig.6 corrected values

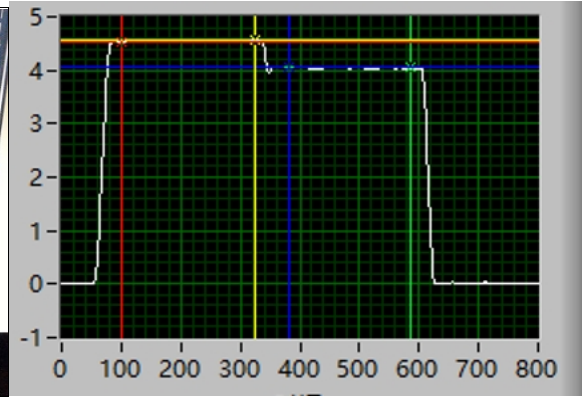

Fig.7 Calibratiion

After the software correction, before the measurement of horizontal linear fluctuations in the laser within $0.040 \mathrm{~mm}$, it can be considered at the track level is qualified on the table 


\section{Calibration}

Before carrying on the test, carries on the calibration to the system. This system calibration procedure is as follows: (1). Secure the sensor and gauge block to the platform. (2). Ten contours were measured on the platform, and the contours were processed in an average value. (3). Place a pair of gauges with a height difference, measure ten times for the same position, and average each group of data. (4). The height of the contour of the gauge block placed afterwards is height-corrected with reference to the contour line when no block is placed. (5). Select and calculate the average height difference between the two sections. (6). Statistical data, and gets the system error data.

In this experiment, six sets of 4-to-4.5mm, 4-5mm, 4-5.5mm, 4-6mm, 4-6.5mm and 4-7mm gauges were calibrated and measured. The error data are shown in Table1.

Table 1 The error date

\begin{tabular}{|l|l|l|l|l|l|l|}
\hline Group & $4-4.5 \mathrm{~mm}$ & $4-5 \mathrm{~mm}$ & $4-5.5 \mathrm{~mm}$ & $4-6 \mathrm{~mm}$ & $4-6.5 \mathrm{~mm}$ & $4-7 \mathrm{~mm}$ \\
\hline $\begin{array}{l}\text { Difference / } \\
\mathrm{mm}\end{array}$ & 0.504224 & 1.00439 & 1.50669 & 2.00486 & 2.51289 & 3.00276 \\
\hline Error & 0.004224 & 0.00439 & 0.00669 & 0.00486 & 0.01289 & 0.00276 \\
\hline
\end{tabular}

\section{Testing and Analysis}

\section{Testing}

the aluminum bottom plate used for placing the foundation is measured, and the results are shown in Fig.8 and its 3Dimage is shown in Fig.9

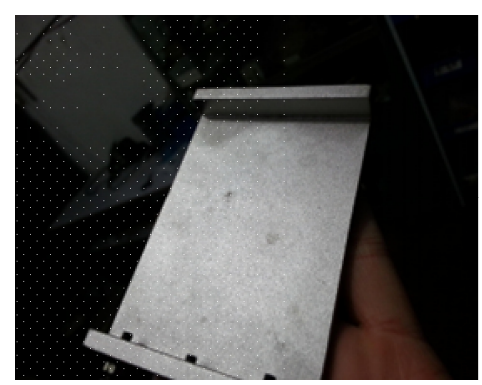

Fig.8 Bottom plate

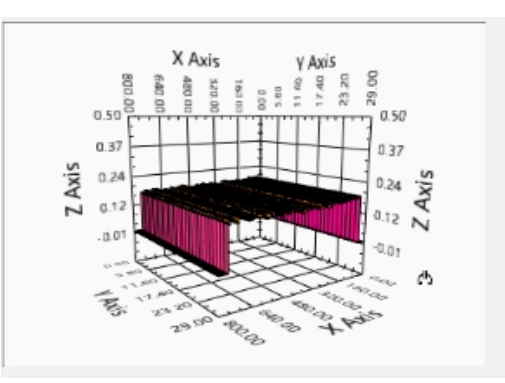

Fig.9 its 3D image

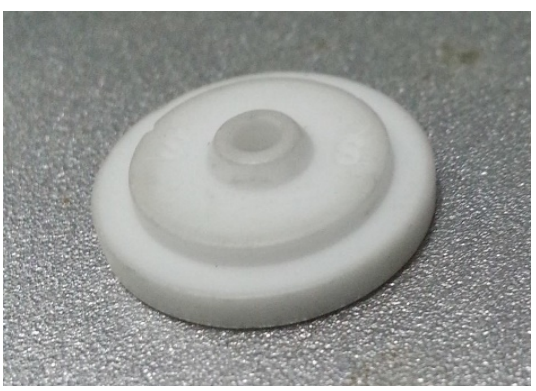

Fig.10 Shaped ceramic - concave up

Then, metal, ceramic and other materials taking measurement experiment, the measurement results of ceramic and metal mobile phone parts are shown in Figure 1011 and Figure 12 13, respectively.
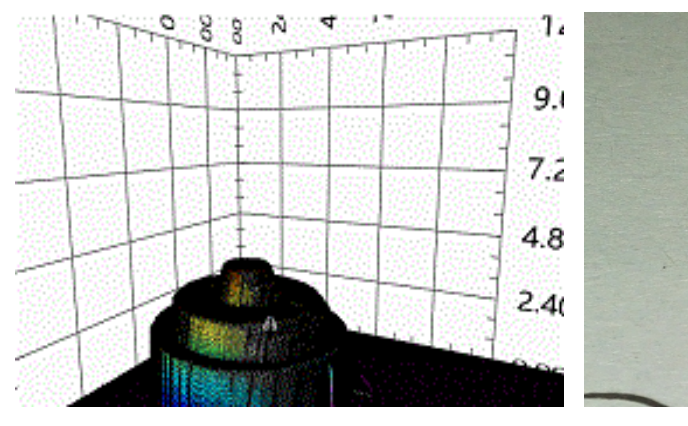

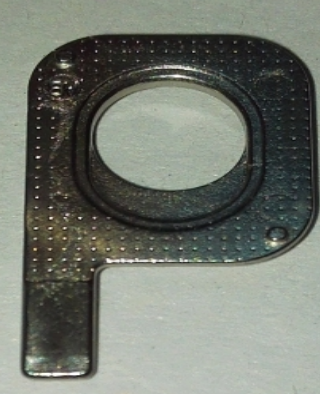

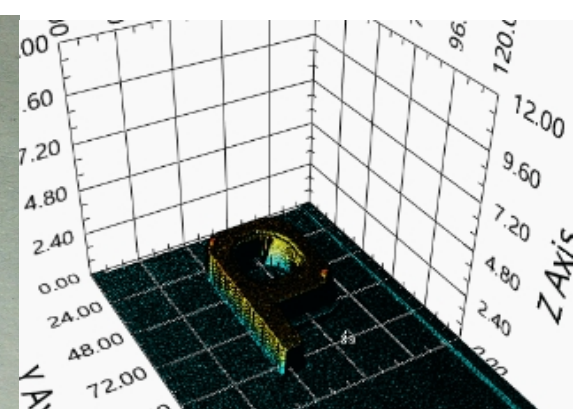




\section{Analysis}

From the experimental process and results, it is first necessary to evaluate whether the measured object can be measured by the contour scanning sensor, and then consider which measurement value and measurement environment to obtain, for example, it is not feasible to determine the dynamic measurement by the above static measurement. But also consider the reflective surface properties of the measured object, the material properties of the measured object and contour defects such as these basic factors. In addition, using the correct filter and the exposure time setting to improve the continuous signal reflected from the contour surface to prevent it into a low utilization of the defect signal, such as measuring a black rubber type of test object, the measured object does not move or Slower movement, longer exposure time may be faster than contour movement to help obtain complete contour information

\section{Conclusions}

In this paper, a 2D measurement system of parts based on 2D laser displacement sensor is designed. The 2D laser sensor is the core component, and the Labview graphical programming software is used as the main software platform. The precise control of the programmable logic controller (PLC) Hardware and software combined with the method of system calibration, and accurate establishment of the corresponding transformation of the relationship between the imaging point and object point, for obtain the measured surface 3D topographic coordinates. Experiments show that the system can achieve high-precision 3D measurement of metal, ceramic and other materials parts, and more automation with lower cost, practical compared with foreign detection systems.

\section{Acknowledgements}

This work was financially supported by the 2016 Free Study Project of Graduate Students of Foshan University ( 2016LGY19 )

\section{References}

[1] Hyojoo Son, Changwan Kim, Kwangnam Choi. Rapid 3D object detection and modeling using range data from 3D range imaging camera for heavy equipment operation[J].

Automation in Construction, 2010,19(2): 898-906.

[2] Li Peng, Liu Qihong. Dimension Measurement of Straight-Cut Gear Based on Machine Vision[J]. Computer Measurement \& Control, 2009.17(4):646 647(in Chinese).

[3] LUO Shu-qian,ZHOU Guo-hong. Medical Image Processing and Analysis [M]. Beijing :Science Press ,

$2003: 140-201$.

[4] Wang Zhongfei, Lin Maosong, Peng Yong, Chu Xiaolong, Yan Fawen. Decting Thickness of Micro Flakes Based on Computer Vision[J].Computer Measurement \& Control 2013 , 21(1):36 38(in Chinese).

[5] ELIAS N, MALAMAS, MICHALIS. A survey on industrial vision system application and tools[J]. Image and vision computing,2007,21:181 188.

[6] Toxqui-Quitl C, Cardenas-Franco J, Padilla-Vivanco A. Bottle inspector based on machine vision[C]. Electronic Imaging. International Society for Optics and Photonics, 2013: 211-213. 
[7] Wang Y, Lv H Y, Wang N. A Machine Vision System for Measurement of Mechanical Parts Based on Lab VIEW 2012[J]. Advanced Materials Research, 2014, 834: 1186-1189.

[8] Huang J, Zhang X B. 3D measuring techniques based on image processing[J].

Chinese Journalof Electron Devices,2002,25(4): 364-368(in Chinese). 